\title{
Analysis of Half Round Runner Dimensions Influence to Plastics Injection Results on Making Door Handle Using Taguchi Method
}

\author{
Ulfa Fairuz Izdihar, Jata Budiman, and Gamawan Ananto
}

\begin{abstract}
Runner is a part of channel systems that flows the molten plastics from the sprue to the gate with a specified layout. The selection of runner shapes are according to the design of the mold and the product. Usually, the diameter of half round runner is calculated by the existing empirical formula. Predictably, the dimension of the runner is one of the factors that will be influencing the injection process and the resulting product. The objectives of experiment are to know how big the runner diameter that will be influencing the taken response and to prove that the existing empirical formula for calculating the diameter of runner is efficient. The experiment is designed by Taguchi method using Minitab applications to generate data of the influences of the runner diameter. The responses of this experiment are cycle time and product mass. The experiment is conducted by using the door handle products with Acrylonitrilebutadiene-styrene as known as ABS material. Besides diameter of runner, there are also other factors from parameters of injection process to be considered, they are nozzle temperature and injection pressure. In reference to Taguchi method, the authors took nine times experiment with three replications or repetitions for each data of experiment. The data from experiment were analyzed and calculated by Analysis of Variance (ANOVA) using Minitab applications to generate the results of experiment. As a result, the diameter of the runner had impact on the responses taken, either to cycle time and product mass as well. In conclusion, the runner diameter was ranked as the most influential compared to nozzle temperature and injection pressure.
\end{abstract}

Keywords-Runner Diameter, Injection Process, Taguchi, Analysis of Variance (ANOVA).

\section{INTRODUCTION}

Injection plastics process is one of manufacturing technologies that is applied by some industries that produce products made from plastics. Injection plastics process is the process to flow molten plastics that is melted at specific melt temperature depends on the kind of plastics that is used for making products. The molten plastics flow to mold with the system which is called channel system. One of the main channel system on injection plastics process is runner system.

Runner system is the channel system that guides the molten plastics from sprue to gate with variant layout of the channel depends on the design of mold and the product

Ulfa Fairuz Izdihar, Jata Budiman, and Gamawan Ananto are with Department of Manufacturing Engineering, Politeknik Manufaktur Negeri Bandung, Bandung, 40135, Indonesia. E-mail: izdihar.fairuz@ gmail.com.

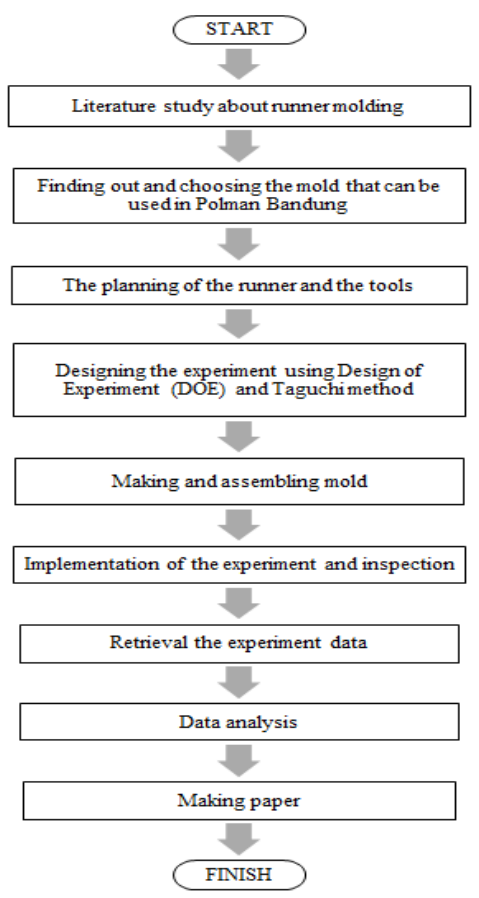

Figure 1. Flowchart of experiment.

specifications. There are several shapes of runner of the mold i.e. full round, half round, square, and trapezoid. The shapes of the runner, especially the half round runner consists of two dimensions. There are diameter and length dimensions. The diameter of half round runner is determined and calculated from the existing empirical formula. The dimensions of runner in diameter and length are expected to influence the setting of injection parameters on injection plastics process. The result of changing the injection parameters that are influenced by dimensions of runner is the product will be forming with variant conditions. Doing some experiments are the steps to find out how big the influences of runner diameter to injection plastics process and products.

This experiment was conducted with the aim of knowing the influence of the change of dimensions of runner against the results of the injection process. This experiment is done by through a case study of the process of making door handle by using the mold which is available at Polman Bandung. To retrieve and analyze data, the Design of Experiment (DOE) designed by Taguchi method and analyzed by Analysis of Variance (ANOVA). This 
experiment is also using Minitab application for generate data and results.

\section{Methodology}

Fig. 1 shows the diagram of flow process (flowchart) that is done.

In this experiment, the tool is the two plate mold which produces door handle. The tool is an inventory of Tool Maker Sector at Bandung Polytechnic for Manufacturing. The construction of two plate mold shown in Fig. 2.

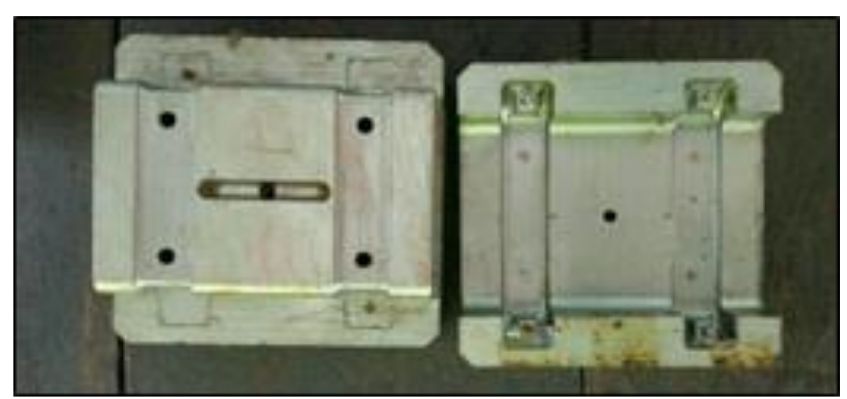

Figure 2. Mold of door handle.

As for the runner, the shape is half-round. The insert runner is assembled into the pocket on the core of mold [1]. The construction of the insert runner is shown in Fig. 3.

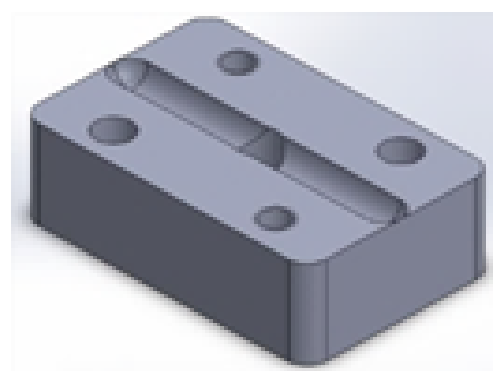

Figure 3. Insert runner.

\section{DESIGN OF EXPERIMENT WITH TAGUCHI METHOD}

The steps taken in the experiments for retrieving data use the Design of Experiment (DOE) with Taguchi method. The steps are as follows.

\section{A. The selection of responses}

The responses chosen in this experiment are cycle time of injection process and mass of product [2].

\section{$B$. The selection of quality characteristics}

There are three characteristics of quality i.e. "Smaller is Better", "Larger is Better", and "Nominal is Best" [3]. Therefore, the characteristic of quality for the response cycle time is based on "Smaller is Better" and also the characteristic of quality for the response mass of products is based on "Nominal is Best".

\section{The selection of factor experiments}

\section{Runner diameter}

Dimensions of runner have the influential effect on the results of the injection process [4]. The dimension that will be used as variable is the diameter of runner. The selection of first runner diameter based on the calculation from existing empirical formula with $S_{\max }$ (maximum thickness of products) $=6 \mathrm{~mm}$ is $7.5 \mathrm{~mm}$ in diameter.

$$
\mathrm{D}=\mathrm{S}_{\max }+1,5 \mathrm{~mm}
$$

To know the influences of runner diameter, $5.5 \mathrm{~mm}$ in diameter is selected to be the smaller diameter than $7.5 \mathrm{~mm}$ and $9.5 \mathrm{~mm}$ in diameter is selected to be the greater diameter than $7.5 \mathrm{~mm}$.

2. Nozzle temperature

Nozzle temperature is the parameter of injection process to set melting point at specific temperature depends on the plastic material [2]. The higher temperatures used in injection process will producing a more fluid texture of molten plastics, so it making easier to entry the mold cavity to form products. Contrary, the lower temperatures, the harder molten plastics to entry the mold cavity. In this study, the plastic material used is Acrylonitrile-butadienestyrene as known as $\mathrm{ABS}$ with range of nozzle temperature ABS revolves around $180^{\circ} \mathrm{C}-230^{\circ} \mathrm{C}$. So, the authors chose the medium range of nozzle temperature i.e. $203^{\circ} \mathrm{C}, 208^{\circ} \mathrm{C}$, and $213^{\circ} \mathrm{C}$.

3. Injection pressure

Injection pressure is the pressure of the injection which is used to push the molten plastic from the nozzle towards the cavity [2]. The higher injection pressure at constant value of the injection speed, the faster time to fill the product. Contrary, the lower injection pressure, the slower time for to fill the product. The injection pressure range for material ABS allowed is $560 \mathrm{kgf} / \mathrm{cm}^{2}-1760 \mathrm{kgf} / \mathrm{cm}^{2}$ (65bar of machine unit - 203bar of machine unit). Meanwhile, the maximum injection pressure of the injection machine is 190bar of machine unit so that the range of the injection pressure that can be used with safety factor required is $65 \mathrm{bar}$ of machine unit $-160 \mathrm{bar}$ of machine unit. Therefore, the authors chose the medium range of injection pressure i.e. 91,94 , and $97 \mathrm{bar}$ of machine unit.

\section{The selection of orthogonal matrix}

Orthogonal matrix is chosen based on a number of factors and the level which were determined before [3]. In accordance with the Taguchi method, orthogonal matrix in this study is shown in Table 1.

TABLE 1.

ORTHOGONAL MATRIX

\begin{tabular}{|c|c|c|c|}
\hline \multirow{2}{*}{$\begin{array}{l}\text { Number of } \\
\text { Experiment }\end{array}$} & \multicolumn{3}{|c|}{ Experiment factors } \\
\hline & $\mathbf{R}$ & NT & IP \\
\hline 1 & 5.5 & 203 & 91 \\
\hline 2 & 5.5 & 208 & 94 \\
\hline 3 & 5.5 & 213 & 97 \\
\hline 4 & 7.5 & 203 & 94 \\
\hline 5 & 7.5 & 208 & 97 \\
\hline 6 & 7.5 & 213 & 91 \\
\hline 7 & 9.5 & 203 & 97 \\
\hline 8 & 9.5 & 208 & 91 \\
\hline 9 & 9.5 & 213 & 94 \\
\hline
\end{tabular}




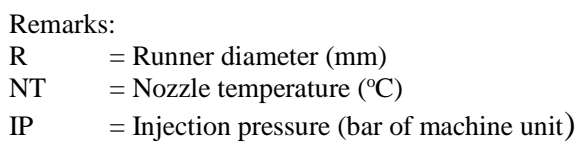

\section{ANALYSIS AND DISCUSSION}

\section{A. Data analysis for cycle time}

Table 2 shows the results of the experiment with the response cycle time [3].

TABLE 2.

EXPERIMENTS RESULTS FOR CYCLE TIME RESPONSE

\begin{tabular}{cccccccc}
\hline \hline \multirow{2}{*}{$\mathbf{R}$} & \multicolumn{3}{c}{$\boldsymbol{N T}$} & \multicolumn{3}{c}{$\boldsymbol{I P}$} & \multicolumn{3}{c}{ Cycle Time $(\mathbf{s})$} \\
\cline { 2 - 8 } & Set & Actual & Set & Actual & $\mathbf{1}$ & $\mathbf{2}$ & $\mathbf{3}$ \\
\hline 5.5 & 203 & 203 & 91 & 91 & 22.41 & 22.38 & 22.56 \\
5.5 & 208 & 208 & 94 & 94 & 22.21 & 22.19 & 22.34 \\
5.5 & 213 & 213 & 97 & 96 & 21.76 & 21.56 & 21.68 \\
7.5 & 203 & 202 & 94 & 94 & 22.98 & 23.01 & 22.50 \\
7.5 & 208 & 208 & 97 & 96 & 22.06 & 22.10 & 22.13 \\
7.5 & 213 & 214 & 91 & 90 & 22.56 & 22.78 & 23.01 \\
9.5 & 203 & 203 & 97 & 96 & 23.02 & 23.04 & 23.14 \\
9.5 & 208 & 208 & 91 & 91 & 23.17 & 23.23 & 23.19 \\
9.5 & 213 & 214 & 94 & 93 & 23.02 & 23.17 & 23.10 \\
\hline \hline
\end{tabular}

From the data in Table 2, data processing is performed using the Taguchi method. According to quality characteristic "Smaller is Better" for the response cycle time, Table 3 displays the main effect of the experiment results.

TABLE 3.

MAIN EFFECT OF CYCLE TIME

\begin{tabular}{ccccc}
\hline \hline Factors & Level 1 & Level 2 & Level 3 & Delta \\
\hline R & -26.895 & -27.070 & -27.280 & 0.38 \\
NT & -27.152 & -27.047 & -27.047 & 0.10 \\
IP & -27.162 & -27.129 & -26.954 & 0.21 \\
\hline \hline
\end{tabular}

The delta value for each experiment factors which are runner diameter, nozzle temperature, and injection pressure successively is $0.38 ; 0.10 ; 0.21$, so that it can be concluded that the diameter of the runner became the most influential factors compared to injection pressure and nozzle temperature.

The next step is calculating the data using the method of variant analysis (ANOVA). The results of the calculations are shown in Table 4.

TABLE 4

ANOVA'S TABLE OF CYCLE TIME

\begin{tabular}{ccccrrr}
\hline \hline Factors & DOF $(\boldsymbol{f})$ & \multicolumn{1}{c}{ S } & \multicolumn{1}{c}{ V } & \multicolumn{1}{c}{ F } & \multicolumn{1}{c}{$\mathbf{S}^{\prime}$} & \multicolumn{1}{c}{ P } \\
\hline A & 2 & 0.222 & 0.111 & 34.80 & 0.216 & $66.33 \%$ \\
B & 2 & 0.022 & 0.011 & 3.44 & 0.016 & $4,80 \%$ \\
C & 2 & 0.075 & 0.037 & 11.71 & 0.068 & $21,02 \%$ \\
Error & 2 & 0.006 & 0.003 & - & - & - \\
Total & 8 & 0.326 & - & - & - & - \\
\hline \hline
\end{tabular}

Remarks :

$\mathrm{S}=$ Sum of squares $\quad \mathrm{S}^{\prime}=$ Pure of sum squares

$\mathrm{V}=$ Mean squares $\quad \mathrm{P}=$ Percent influence

$\mathrm{F}=\mathrm{F}$-ratio

The graph on Fig. 4 on the next page shows the percentage of the experiment factors influence the response cycle time.

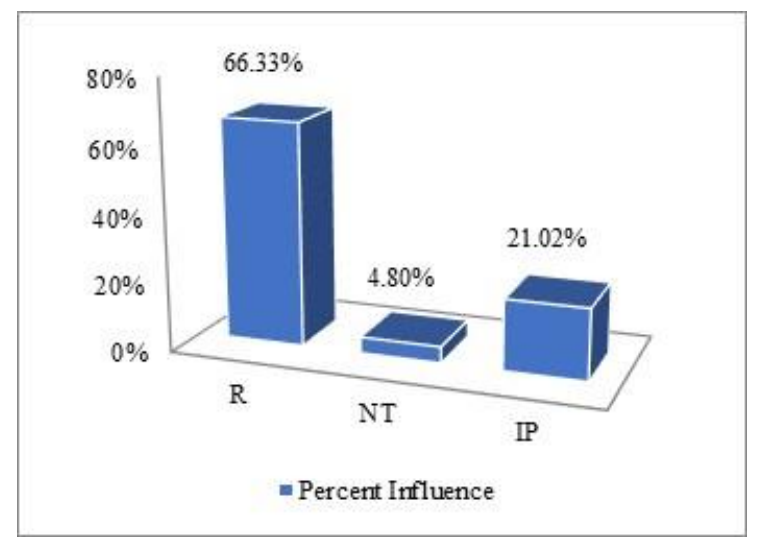

Figure 4. Percent influence of each experiment factors for cycle time.

From the results, it was known that the diameter of the runner had the most influence than other factors. From the results shown in Fig. 4., it can be concluded that the diameter of the runner will not affect how fast or how long the cycle time is generated because other factors i.e. injection pressure and nozzle temperature also have an effect on the cycle time. The percentage of the results can still change because there are still other internal (from injection machine system) and external factors (from environmental factors) that cannot be set and have an impact on the results of injection process.

\section{B. Data analysis of mass of products}

The results of the experiment with the response cycle time are shown in Table 5 [3].

TABLE 5.

EXPERIMENT RESULTS FOR MASS OF PRODUCTS RESPONSE

\begin{tabular}{cccccccc}
\hline \hline \multirow{2}{*}{$\mathbf{R}$} & \multicolumn{3}{c}{$\boldsymbol{N T}$} & \multicolumn{3}{c}{$\boldsymbol{I P}$} & \multicolumn{3}{c}{ Mass of products (g) } \\
\cline { 2 - 8 } & Set & Actual & Set & Actual & $\mathbf{1}$ & $\mathbf{2}$ & $\mathbf{3}$ \\
\hline 5,5 & 203 & 203 & 91 & 91 & 18,00 & 18,19 & 17,96 \\
5,5 & 208 & 208 & 94 & 94 & 18,02 & 18,09 & 18,04 \\
5,5 & 213 & 213 & 97 & 96 & 18,14 & 18,17 & 18,10 \\
7,5 & 203 & 202 & 94 & 94 & 18,34 & 18,31 & 18,14 \\
7,5 & 208 & 208 & 97 & 96 & 18,58 & 18,11 & 18,11 \\
7,5 & 213 & 214 & 91 & 90 & 18,56 & 18,48 & 18,57 \\
9,5 & 203 & 203 & 97 & 96 & 18,53 & 18,46 & 18,52 \\
9,5 & 208 & 208 & 91 & 91 & 18,31 & 18,56 & 18,56 \\
9,5 & 213 & 214 & 94 & 93 & 18,27 & 18,27 & 18,45 \\
\hline \hline
\end{tabular}

From the data in Table 5, data processing is performed using the Taguchi method. According to characteristic quality "Nominal is Best" for the response mass of product with $18.35 \mathrm{~g}$ as the appropriate product mass, Table 4 displays the main effect of the experiment results.

TABLE 6.

MAIN EFFECT OF MASS OF PRODUCTS

\begin{tabular}{ccccc}
\hline \hline Factors & Level 1 & Level 2 & Level 3 & Delta \\
\hline R & 34.634 & 38.534 & 41.068 & 6.434 \\
NT & 38.232 & 36.194 & 39.809 & 3.615 \\
IP & 36.719 & 40.037 & 37.479 & 3.318 \\
\hline \hline
\end{tabular}

The delta value for each experiment factors which are runner diameter, nozzle temperature, and injection pressure 
successively is $6.434 ; 3.615 ; 3.318$, so that it can be concluded that the diameter of the runner became the most influential factors compared to injection pressure and nozzle temperature.

The next step is calculating the data using the method of variant analysis (ANOVA). The results of the calculations are shown in Table 7.

TABLE 7.

ANOVA'S TABLE OF MASS OF PRODUCTS

\begin{tabular}{ccrrrrr}
\hline \hline Factors & DOF $(\boldsymbol{f})$ & \multicolumn{1}{c}{ S } & \multicolumn{1}{c}{ V } & \multicolumn{1}{c}{ F } & \multicolumn{1}{c}{$\mathbf{S}^{\prime}$} & \multicolumn{1}{c}{ P } \\
\hline A & 2 & 63,021 & 31,511 & 6,421 & 56,822 & $54,09 \%$ \\
B & 2 & 19,705 & 9,852 & 1,561 & 5,877 & $13,36 \%$ \\
C & 2 & 18,135 & 9,067 & 2,686 & 17,670 & $11,89 \%$ \\
Error & 2 & 5,491 & 2,746 & - & - & - \\
Total & 8 & 106,353 & - & - & - & -
\end{tabular}

Remarks :

$\mathrm{S}=$ Sum of squares $\quad \mathrm{S}^{\prime}=$ Pure of sum squares

$\mathrm{V}=$ Mean squares $\quad \mathrm{P}=$ Percent influence

$\mathrm{F}=$ F-ratio

The graph on Fig. 5 shows the percentage of the experiment factors influence the response mass of products.

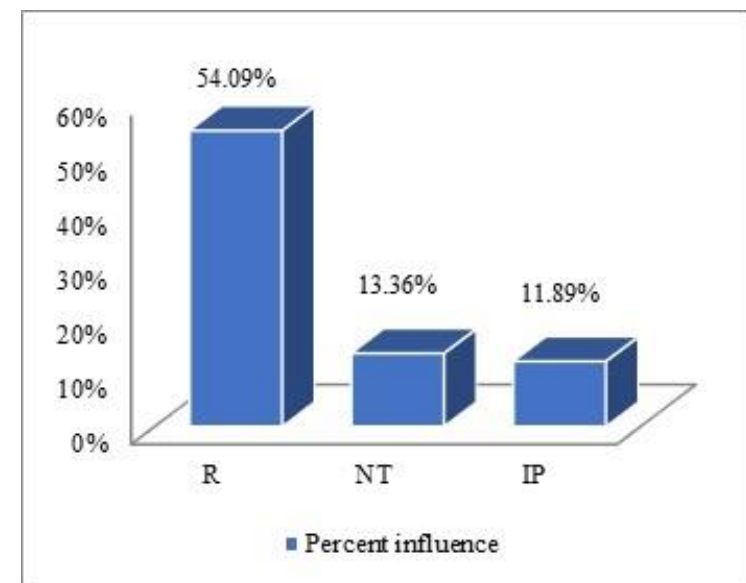

Figure 5. Percent influence of each experiment factors for mass of products.

Fig. 5. shows that the dimension of the runner has the most influence than other factors. The diameter of the runner has the influence on the mass of the product because the mass of the resulting product are various. It can be concluded when the diameter of the runner used is smaller

than the diameter of the runner from existing empirical formula, the products will be increasingly lighter. It might be showed by the sink mark from injection results. Contrary, the greater runner diameter than the diameter of the runner from existing empirical formula, the heavier products. The results fit with the existing theory, the smaller the cross-section runner used, then temperatures occur in the runner will be higher than in the barrel, so it increases the potential of sink mark form on the product and it causes the resulting product lighter.

The percentage of the results can still change because there are still other internal (from injection machine system) and external factors (from environmental factors) that can not be set and have an impact on the results of injection process.

\section{CONCLUSIONS}

From the results of experiment, the conclusions can be withdrawn. They are as follows:

1) The runner dimensions are various, especially runner diameter, affects the response taken i.e. the cycle time and the mass of the product.

2) In term of response cycle time, it can be concluded that the larger diameter of the runner, the longer time required to fill the cavity. The smaller diameter of the runner, the shorter time required to fill the cavity.

3) Regarding the product mass response, if the diameter of the runner used is smaller than its calculation then the mass of product will be reduced. On the other hand, as the diameter of the runner used is larger than its calculation, the resulting product mass will be increased, e.g. it might be shown by its sink mark on the product that is visually less.

\section{REFERENCES}

G. Menges, W. Michaeli, and P. Mohren, How to Make Injection Molds, 3rd ed. Munich: Carl Hanser Verlag, 2000.

H. Rees, Mold Engineering. Munich: Carl Hanser Verlag, 2002.

R. Roy, Design of experiments using the Taguchi approach: 16 steps to product and process improvement. New York: John Wiley \& Sons Inc, 2001.

Budiarto, Perancangan Peralatan Pencetak. Bandung: Polman, 2002. 